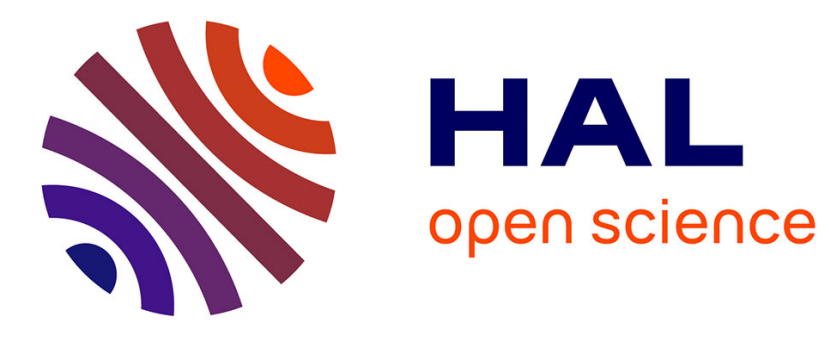

\title{
L'agriculture uruguayenne face aux investisseurs sud-américains
}

Alejandro Saravia, Martine Guibert, Pedro Arbeletche, Maëlle Gedouin, Luc Capdevila, Hermes Moralès, Jean-François Tourrand

\section{- To cite this version:}

Alejandro Saravia, Martine Guibert, Pedro Arbeletche, Maëlle Gedouin, Luc Capdevila, et al.. L'agriculture uruguayenne face aux investisseurs sud-américains. Autrepart - Revue de sciences sociales au Sud, 2015. hal-02081997

\section{HAL Id: hal-02081997 \\ https://hal-univ-tlse2.archives-ouvertes.fr/hal-02081997}

Submitted on 2 Apr 2019

HAL is a multi-disciplinary open access archive for the deposit and dissemination of scientific research documents, whether they are published or not. The documents may come from teaching and research institutions in France or abroad, or from public or private research centers.
L'archive ouverte pluridisciplinaire HAL, est destinée au dépôt et à la diffusion de documents scientifiques de niveau recherche, publiés ou non, émanant des établissements d'enseignement et de recherche français ou étrangers, des laboratoires publics ou privés. 


\title{
L'agriculture uruguayenne face aux investisseurs sud-américains
}

\author{
Alejandro Saravia*, Martine Guibert**, Pedro Arbeletche***, \\ Maëlle Gédouin****, Luc Capdevila*****, Hermes Morales ******, \\ Jean-François Tourrand*******
}

En Uruguay, pays qui s'est construit autour des élevages bovin et ovin à partir du XVII siècle, la surface agricole totale de 16 millions d'hectares était consacrée en grande partie à cette activité extensive. Or, à la fin $\mathrm{XX}^{\mathrm{e}}$ siècle et surtout au début du $\mathrm{XXI}^{\mathrm{e}}$ siècle, le pays a connu un développement accéléré des productions végétales, directement lié à l'installation d'acteurs étrangers. Depuis plusieurs décennies, les riziculteurs brésiliens du Rio Grande do Sul mettent en valeur les terres de l'Est et le mouvement tend à s'amplifier. Depuis les années 1990, des plantations sylvicoles (eucalyptus et pins) ont été déployées sur plus d'un million d'hectares; elles sont la propriété de quelques grands groupes d'origine nord-européenne (Finlande), étatsunienne, chilienne. Plus récemment, au cours de la décennie 2000, le modèle argentin de production de soja s'est implanté dans l'Ouest du pays; en 2014, il occupe 1,3 million d'hectares. D'ici 2020, la cellulose pourrait devenir le premier produit exporté, devançant la viande bovine qui représentait $70 \%$ des exportations en valeur au début des années 2000 et qui a été dépassée pendant un an par le soja.

Alors que les investissements dans le secteur agricole et agro-industriel uruguayen étaient d'origine européenne et étatsunienne, ils sont, au début des années 2000, le fait d'acteurs argentins et brésiliens, et secondairement chiliens. La situation de l'Uruguay est commune à l'ensemble des pays de la région, avec des variantes liées aux contextes macro-économiques et aux réglementations nationales (fiscales, environnementales). Cependant, en raison de la taille réduite de son agriculture, les impacts sont amplifiés : dès lors, que devient le producteur

\footnotetext{
* Vétérinaire, Instituto Plan Agropecuario (IPA), Uruguay.

** Géographe, UMR CNRS 5193 LISST/Dynamiques rurales, université Toulouse - Jean Jaurès.

*** Ingénieur agronome, facultad d'agronomie, université de la République (UDELAR), Uruguay.

**** Agro-économiste, doctorante, AgroParisTech.

****** Historien, université de Rennes 2.

******* Ingénieur agronome, Instituto Plan Agropecuario (IPA), Uruguay.

******** Vétérinaire, Cirad-Green\&MAAF, France.
} 
gaucho de la Pampa uruguayenne avec son troupeau de bovins et d'ovins, sa petite production de céréales pour sa consommation, l'alimentation d'appoint de ses animaux et la vente des excédents ? Qu'en est-il des bosquets pour la production de bois (construction, outillage, cuisine et chauffage) ? Quelles transformations affectent la société rurale dans ses fonctionnements et dans son maintien ?

Les projets de recherche PIC (1 et 2), MOUVE (ANR/Systerra) et ANR/Agrifirme ont permis la réalisation de travaux de terrain en Uruguay entre 2011et 2013, avec plusieurs dizaines d'entretiens formels et informels effectués. En croisant ces matériaux avec le suivi de la réalité agricole et rurale, et avec des données secondaires, nous privilégions une approche qualitative, l'accès à des informations quantitatives demandant un approfondissement qui n'a pas encore été mené à bien. Ầ ce stade, nous pouvons rendre compte des facteurs d'installation en Uruguay d'acteurs sud-américains de l'agrobusiness et des impacts sur l'agriculture et les sociétés locales.

Nous rappelons tout d'abord les faits marquants de l'intégration historique de l'Uruguay agricole dans la globalisation, en mettant en exergue l'originalité des dernières années, liée aux investissements sud-américains. Nous exposons ensuite les modalités d'implantation des entreprises venues des pays voisins et leur participation aux mutations de l'agriculture uruguayenne. Nous donnons des éléments qualitatifs sur leurs identités et leurs caractéristiques. Nous discutons enfin les conséquences pour les espaces ruraux.

\section{L'Uruguay, le petit pays sud-américain entré précocement dans la globalisation}

$\mathrm{Au} \mathrm{XIX}^{\mathrm{e}}$ siècle, l'Uruguay est connu pour ses exportations de cuirs et de peaux, surtout vers l'Europe. Il est aussi le principal fournisseur de viande séchée destinée à la main-d'œuvre des pays cotonniers et sucriers (États-Unis, Brésil, Caraïbe) [Litre, 2010 ; Moraes, 2008 ; Morales et al., 2011]. Un saut est franchi avec le partenariat anglais (capitaux)/allemand (technique) qui permet le développement, à partir de 1865, à Fray Bentos (ville sur le fleuve Uruguay), de l'abattoir frigorifique Liebig's Extract of Meat Company (LEMCO), en lieu et place du saladero (abattoir traditionnel produisant de la viande, éventuellement salée), afin de produire à grande échelle de l'extrait de viande (bouillon cube Oxo), puis du cornedbeef. Penser un bien agro-industriel à partir d'une production locale pour le commercialiser à l'échelle globale est, à cette époque, le grand défi. L'Uruguay s'affirme alors sur le marché mondial aux côtés du voisin argentin.

Lors de la Première Guerre mondiale, le besoin en aliments et en vêtements de l'Europe est un facteur de croissance des exportations et de reconnaissance internationale. Si le corned-beef est le produit phare, des mégisseries européennes fonctionnent déjà avec la laine et les peaux sud-américaines. Mais la crise des années 1930 est rude pour l'Argentine et l'Uruguay, devenus entre-temps de grands producteurs de blé et d'autres grains. 
Les soubresauts de l'économie mondiale, les périodes fastes, plutôt de courte durée, étant suivies par des crises plus ou moins longues, affectent l'Uruguay [Morales, 2007 ; Barrán, Nahum, 1967-1977 ; Finch, 1980 ; Garavaglia, 2000]. Cette alternance s'inscrit dans la durée historique et est un élément-clé de compréhension des stratégies d'adaptation du monde rural, issu, comme le reste de la population uruguayenne, de la colonisation européenne. La période actuelle débutant dans les années 2000 jusqu'au début des années 2010, favorable, est atypique, car longue. Depuis 2008, le prix de la viande de bœuf est en effet élevé, même s'il fluctue. La situation est similaire pour les autres produits agricoles, la demande internationale étant dynamique. Dans des entretiens menés en 2011, encore année de croissance économique, plusieurs acteurs locaux s'inquiétaient de la longueur de cette période bénéfique. Si les jeunes l'apprécient, les plus âgés attendent presque la crise, comme pour se rassurer sur la pérennité des cycles.

En ce qui concerne la présence d'acteurs étrangers, dans les années 1950, la modernisation de l'agriculture en Uruguay et l'adoption de nouveaux systèmes de production découlent des recommandations d'une mission de la FAO [BIRF-FAO, 1951]. L'Uruguay se tourne alors vers la Nouvelle-Zélande. Localisé sous les mêmes latitudes que l'Uruguay, ce pays a des cheptels bovin et ovin comparables. Cependant, l'écosystème naturel de la Nouvelle-Zélande n'est pas la savane, mais la forêt. Ce pays est moins proche de l'Uruguay que de l'Amazonie orientale brésilienne, où a eu lieu, avec un demi-siècle d'écart, un processus comparable de remplacement de la forêt par des pâturages. Des pâturages sont donc implantés à la place des parcours naturels dans la Pampa uruguayenne (aujourd'hui dénommée officiellement Campos [Batello et al., 2011] et sous-entendu, naturales). Le résultat attendu est l'augmentation de la productivité du cheptel en lien avec l'amélioration de son alimentation. C'était compter sans les sécheresses, fréquentes en été comme en hiver, et faire fi des savoirs locaux. De fait, les premiers directeurs du Plan agropecuario, l'agence uruguayenne en charge de la modernisation ${ }^{1}$ (sa mission encore en 2016), étaient des techniciens néo-zélandais ou australiens ne parlant pas castillan. Or, les éleveurs uruguayens, notamment dans le Nord et l'Ouest du pays, disposaient déjà de systèmes de naissage, reélevage et embouche herbagère adaptés aux Campos [Morales, 2007]. La soif de modernité venant de l'étranger a ignoré le potentiel de mise en valeur des savoirs locaux et des écosystèmes naturels.

Le dernier tiers du XX $\mathrm{X}^{\mathrm{e}}$ siècle est marqué en Amérique du Sud par une succession de graves crises politiques, économiques et sociales, avec une période très critique au tournant du millénaire. L'Uruguay est frappé lui aussi, après avoir vécu à la fín des années 1980 les crises de la laine et la dollarisation de l'économie, le tout dans un contexte de post-dictature (fin en 1985). Entre autres conséquences, de nombreux petits producteurs ont été quasiment expulsés de leurs terres, la conversion en dollar de leur dette et la chute des prix agricoles rendant insurmontable un endettement pourtant lié aux investissements réalisés avec l'aval de la profession et l'appui des banques.

1. L'Instituto Plan Agropecuario a pris le relais en 1996. 
Ce bref aperçu historique rappelle l'omniprésence de la globalisation dans le monde rural uruguayen, avec l'inscription précoce de ses productions agricoles dans les marchés internationaux et l'alternance entre des crises longues et sévères, et des périodes courtes et propices.

\section{L'intérêt actuel des investisseurs du Sud et leur rôle dans le boom de l'agriculture uruguayenne}

Deux facteurs ont un rôle majeur dans le boom actuel des productions végétales et animales en Uruguay: la forte demande internationale en commodities et l'intérêt pour l'agriculture de la part d'investisseurs étrangers, sud-américains notamment. Les flux accrus sur le marché mondial résultent en grande partie de l'émergence des marchés asiatiques (Chine). Les pays exportateurs offrent des biens agricoles standardisés dont l'utilisation aval est diverse : biens agricoles bruts, aliments, fibres, biocarburants. Ces flex crops sont prisées par les acteurs agro-industriels globalisés, qui orientent en amont les méthodes de la production agricole en proposant intrants, semences et innovations technologiques, et qui contrôlent en aval les process agroalimentaires.

Dans ce contexte, les investissements réalisés par des acteurs argentins et brésiliens ont été le fer-de-lance d'une modernisation et d'une intensification accrue de la production uruguayenne. Tandis que les entreprises agricoles argentines ont bouleversé les modes de production en grandes cultures, les entreprises agroindustrielles brésiliennes ont redéfini la gouvernance des filières viande et riz, et les entreprises chiliennes, aux côtés des entreprises étatsuniennes et européennes, ont organisé la filière eucalyptus. Or, l'arrivée de ces investisseurs est à mettre en relation avec les conditions forgées par les autorités uruguayennes.

\section{Un contexte national favorable aux investissements}

Dans les années 2000, l'Uruguay devient très attractif pour les investissements directs étrangers (IDE), dont le stock passe de plus de 1,4 milliard de dollars en 2002 à plus de 17,5 en 2012. Les flux d'IDE atteignent 416 millions de dollars en 2003 , se replient en 2004 , puis entament une progression fulgurante : près de 1,5 milliard en 2006, plus de 2,6 en 2012, et près de 2,8 en 2013 [Uruguay XXI, 2014]. La situation est inédite. En plus du secteur de la construction, une grande partie de ces investissements étrangers concerne le secteur primaire, avec la reprise d'exploitations agricoles et la construction ou la modernisation d'unités agroindustrielles, provoquant une hausse de la production et de la productivité. Par exemple, la construction de l'usine de fabrication de cellulose de Botnia, devenue UPM et qui est une entreprise sylvicole à capitaux finlandais, a coûté, entre 2005 et 2006, 1,2 milliard de dollars.

Quant aux pays d'origine des IDE, en 2012, l'Argentine est en tête avec 36,5\% du total (secteurs immobilier et agriculture), loin devant le Brésil (8,5\% dans les secteurs de l'agro-industrie, de la chimie et des plastiques), l'Espagne (5,3\% dans 
les domaines des infrastructures, des banques et des services) et les États-Unis (4,7\% dans les services et l'industrie). Le Chili, avec 0,7\% des IDE, vise les secteurs du commerce de détail et des loisirs [Uruguay XXI, 2014], sachant qu'il a déjà investi les années antérieures, dans des plantations d'eucalyptus.

Des conditions favorables dotent l'Uruguay d'atouts importants. La Loi des investissements $n^{\circ} 16906$ de 1998 constitue un cadre solide : elle déclare d'intérêt national la promotion et la protection des initiatives locales et étrangères. Les investissements étrangers peuvent être réalisés dans toutes les activités et dans les mêmes conditions que celles dont bénéficient les investissements locaux. Le traitement fiscal, en particulier, est similaire et parfois même plus favorable. C'est le cas des zones franches dans les ports (et dans la ville de Montevideo), qui bénéficient d'une exonération fiscale intégrale.

D'autres éléments plaident en faveur de l'Uruguay, à commencer par la localisation géographique stratégique du pays au cœur de la zone Mercosur ${ }^{2}$, le bon développement relatif de ses infrastructures de transport et de télécommunications, et le niveau d'éducation de sa population. Sa stabilité économique est renforcée par des coûts salariaux compétitifs et la disponibilité d'une main-d'œuvre apte à être formée si nécessaire. L'État fait donc le pari du développement des IDE pour qu'ils entraînent l'économie et l'insèrent dans la globalisation. Les politiques publiques créent ainsi « un climat d'affaires » lié au processus d'ouverture, de dérégulation et d'investissements transnationaux.

Dans le secteur agricole, les bonnes conditions sanitaires tant en production végétale qu'animale, l'autorisation des organismes génétiquement modifiés (soja) et les subventions à l'achat de plants d'eucalyptus sont autant de critères qui engendrent des changements structurels voulus par l'État, mais difficilement réversibles en cas de réorientation de cette politique. L'afflux de ces investissements directs étrangers permet d'améliorer la valorisation de ressources sous-utilisées comme des sols fertiles, des infrastructures portuaires, des unités de stockage, etc. Les ressources humaines disponibles (agronomes, techniciens) sont embauchées dans les entreprises. Enfin, la pression fiscale ne représente qu'entre $6 \%$ et $8 \%$ du chiffre d'affaires des entreprises agricoles [Tambler, 2014], un taux intéressant pour les acteurs argentins soumis à une taxation plus élevée dans leur pays.

Dès lors, ce contexte favorise des changements rapides dans le secteur agricole, en lien étroit avec les investissements réalisés par les producteurs argentins, les entreprises sylvicoles et la demande en animaux des abattoirs brésiliens. L’intensification de la production agricole caractérise la période [Errea et al., 2011 ; Ernst, Siri-Prieto, 2012 ; Vassallo, 2012].

2. Marché commun du Sud, entre l'Argentine, le Brésil, le Paraguay et l'Uruguay entré en vigueur fin 1994, et étendu par la suite au Chili et à la Bolivie (1996), au Pérou (2003), à la Colombie et à l'Équateur (2004), et au Venezuela (2012). 


\section{La production uruguayenne de viande de bœuf : abattoirs-conditionneurs brésiliens et remontée de filière}

La demande mondiale est dynamique pour les produits des filières animaux (viande, lait) auxquels accèdent les nouvelles classes moyennes éprises de consommation. Peu de pays au monde ont été capables de développer significativement en peu de temps leur production de viande de bœuf. Le Brésil l'a fait, d'une part, pour la viande de bœuf en transformant en pâturage d'immenses étendues en Amazonie et dans les Cerrados, et, d'autre part, en développant la production intégrée de viandes de volaille et de porcs à partir du maïs et du soja, récoltés dans le pays. Il est devenu en quelques années le premier exportateur mondial de viande (rouge et blanche). D'autres pays ont, comme lui, augmenté la productivité par hectare et/ou par animal : l'Australie, la Nouvelle-Zélande et l'Uruguay. En Uruguay, durant les dernières décennies, l'élevage ovin a cédé la place à l'élevage bovin et les pâturages implantés se sont généralisés. Repoussés par les productions végétales, les troupeaux sont désormais plus nombreux au Nord du pays (Départements d'Artigas et de Salto) et à l'Ouest (Cerro Largo et Treinta y Tres) (carte 1).

Carte 1 - Nombre de bovins (2013) et variation par département (1990-2013)

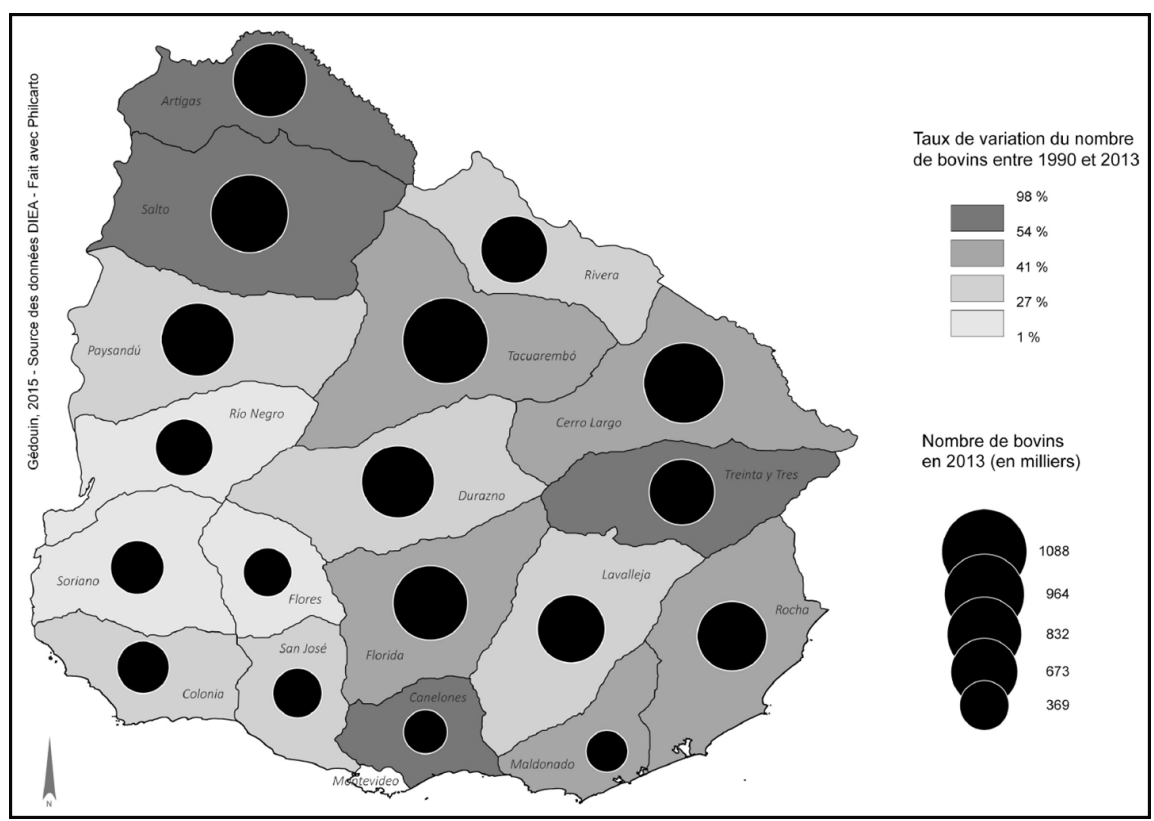

Source : carte élaborée par les auteurs.

Le nombre total de bovins a crû de 10 à 12 millions de têtes entre 2002 et 2010. Il se maintient à ce niveau malgré le déstockage important en lien avec des prix en forte hausse (figure 1) : plus de 4044 dollars la tonne en 2014 contre 2300 en 2005, avec un pic à plus de 4864 en 2011 [Institut Plan Agropecuario (IPA) sur la base de données de l'Institut National des viandes (INAC), 2014]. 
Ces dernières années, des feed lots, unités d'engraissement de plusieurs centaines de jeunes bovins à quelques dizaines de milliers [Saravia et al., 2014], sont installés dans les exploitations. La fabrication d'aliments composés favorise la valorisation des grains produits sur la ferme. Certains producteurs, également, misent à nouveau sur l'embouche herbagère.

Alors que les éleveurs ont modernisé la conduite des troupeaux, avec plus ou moins de réussite et de conviction, la présence croissante d'IDE est flagrante côté transformation, soit en aval de la filière. L'acquisition d'abattoirs-conditionneurs (frigorifiques) a été le fait d'entreprises d'origine brésilienne, surtout en 2006 et 2007 [Bittencourt et al., 2009 ; Bittencourt, Carracelas, Reig, 2011]. Elle concerne les unités les plus modernes, certaines étant déjà tournées vers des niches de marché (produits carnés élaborés). Sur les trente-quatre frigorifiques existants en Uruguay, dix concentrent à eux seuls $62 \%$ de l'abattage et $82 \%$ des exportations. Sept sont Brésiliens avec trois d'entre eux, Marfrig, JBS (numéro un mondial) et Minerva Foods, qui contrôlent $40 \%$ de l'activité et $50 \%$ des ventes uruguayennes de viande de bœuf. Récemment des restructurations importantes ont eu lieu : cession du département viande de bœuf de Brasil Foods, entrée d'intérêts chinois dans la composition du capital de Minerva Foods [El Observador, 2012, 2013]. Ultime nouveauté en Uruguay de ces années 2010 : l'industrie frigorifique intègre la production primaire à travers l'installation et le contrôle de feed lots.

Deux tiers de la production de viande de bœuf sont écoulés à l'export, le tiers restant étant donc consommé en interne. Les figures 1 et 2 montrent les évolutions de ces exportations et leurs destinations. Le changement est net depuis le début de la décennie 2000 : le marché nord-américain s’estompe au profit des marchés asiatiques.

Figure 1 - Évolution des exportations de viande de bœuf de l’Uruguay entre 2001 et 2013

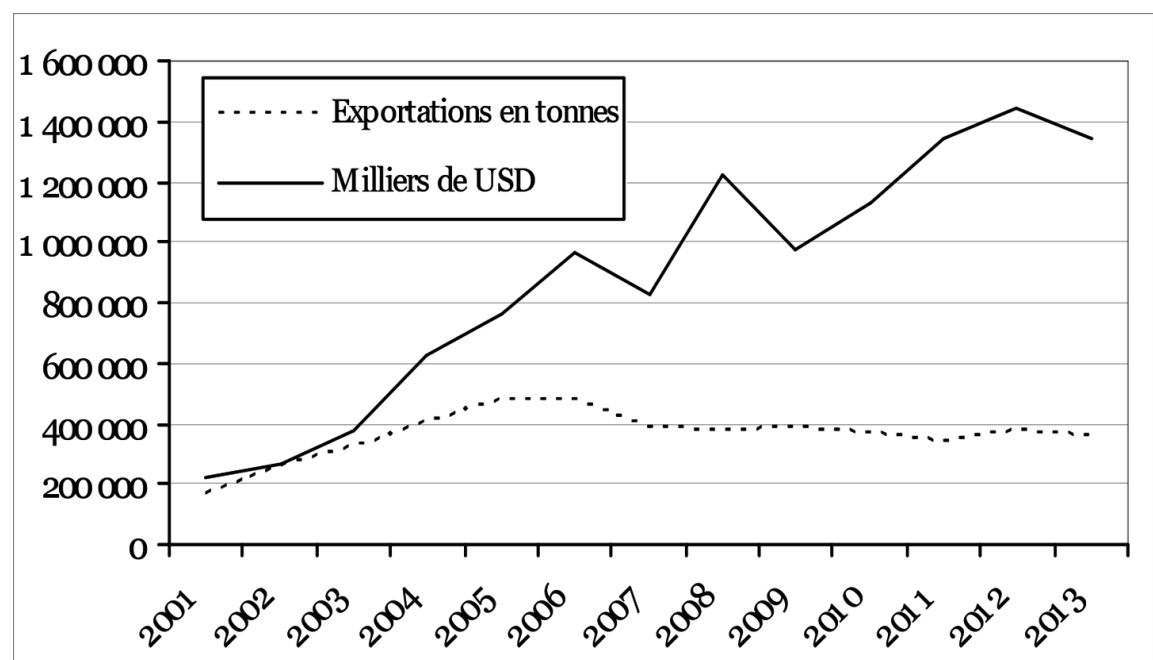

Source : ministère de l'Agriculture et de l'Élevage uruguayen - Direction des statistiques (MGAP-DIEA). 
Figure 2 - Évolution par destination entre 2001 et 2013 des exportations de viande de bœuf de l'Uruguay (en tonnes)

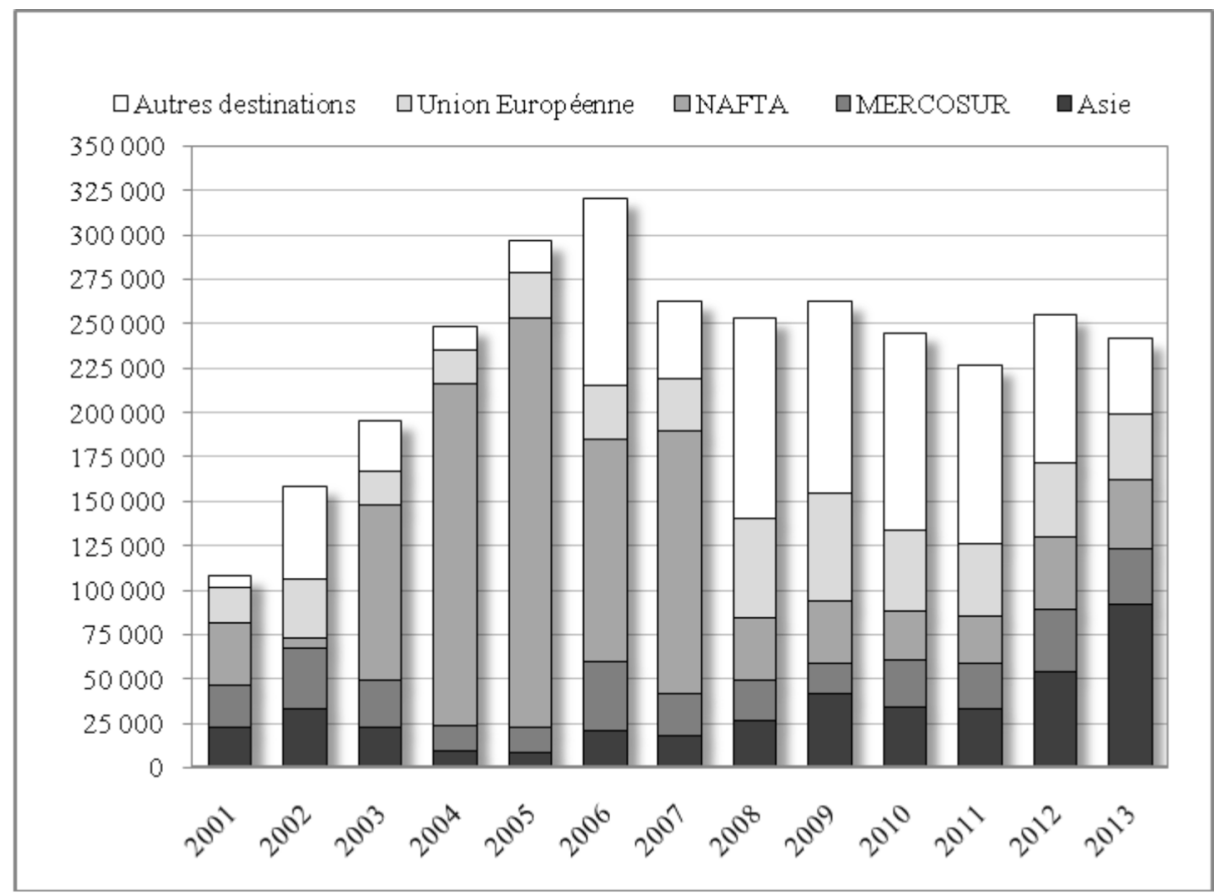

Source : ministère de l'Agriculture et de l'Élevage uruguayen - Direction des statistiques (MGAP-DIEA).

Une précision d'ordre socioculturel peut être apportée : le développement de l'agriculture brésilienne et de l'élevage [Buainain et al., 2014] sur les terres amazoniennes et des Cerrados, est animé par des producteurs du Rio Grande do Sul, appelés eux aussi Gauchos, qui ont migré et ont prospéré. Il est fréquent qu'un Gaucho, descendant ou assimilé, soit à la tête des grands groupes brésiliens précédemment cités [Tourrand et al., 2013]. Autrement dit, la réputation de l'élevage gaucho uruguayen a attiré l'agro-industriel gaucho brésilien, d'autant plus qu'il existe une forte complémentarité entre le Sud du Brésil et l'Uruguay en termes de type et de qualité de la viande.

\section{Les grandes cultures : « los argentinos » et la production de soja}

À partir de la fin des années 1990 se sont multipliées en Argentine des formes associatives de production appelées pool de siembra (pool de semis) à la durée variable, parfois le temps d'une ou deux campagnes agricoles (sachant qu'elles avaient déjà commencé dans les années 1980, voire 1970). D’abord organisés entre voisins, les pools consistent à réunir du foncier et du capital, à sous-traiter les travaux (semis direct-pulvérisation-récolte), le tout sur les conseils d'un ingénieur agronome et grâce à une gestion de l'ensemble garantie par des contrats ou des 
formes juridiques élaborées (fidéicommis). La grande diversité de la taille (quelques centaines d'hectares à plusieurs dizaines de milliers), de la composition (entre personnes connues ou pools d'actionnaires extra-agricoles et étrangers), des formes de sous-traitance et des types de contrat oblige à distinguer les pools en fonction de leurs objectifs, de leur évolution et de leur taille économique [Bisang, Anlló, Campi, 2010]. Les plus grands, dits méga-entreprises, opèrent sur les marchés financiers internationaux et captent des fonds d'investissement, des fonds de pension ou des capitaux en mal de placement depuis la crise financière de 2007-2008. Ils sont attirés par le secteur agricole, du fait des retours élevés et rapides sur investissement (les acteurs rencontrés en 2013 parlent de 12 à $15 \%$ par an).

Sur un plan productif, le développement des activités agricoles par ces associations d'acteurs repose sur des itinéraires techniques simplifiés. Le semis direct (ou zéro labour) est privilégié, le maintien des restes d'une récolte favorisant l'activité biologique, limitant l'érosion éolienne et celle liée à la battance des pluies. Il est combiné avec l'emploi de semences génétiquement modifiées. Le coût en machinisme est réduit, le labour ayant toujours été assimilé à une forte consommation d'énergie. En revanche, il est nécessaire de contrôler les adventices grâce à des herbicides sélectifs à l'action prolongée dans le sol. En raison de son rapport coût de production/valorisation commerciale, le soja est la culture privilégiée.

Parmi les acteurs argentins qui franchissent la frontière, surtout à partir du début des années 2000 [Arbeletche, Carballo, 2010 ; Guibert et al., 2011], figurent des méga-entreprises locataires de plusieurs dizaines de milliers d'hectares, ou parfois propriétaires d'une partie des terres, travaillées dans plusieurs pays de l'Amérique du Sud, et fonctionnant en réseau, c'est-à-dire selon un système horizontal d'acteurs associés dans la gestion optimisée des actifs [Arbeletche, Coppola, Paladino, 2012 ; Arbeletche, Gutierrez, 2010 ; Guibert, 2013 ; Clasadonte et al., 2013 ; Requier-Desjardins, Guibert, Bühler, 2014]. Depuis peu, beaucoup de ces «argentinos » se sont retirés suite à des difficultés de retour sur investissement (méconnaissance des conditions locales, mise en culture de terres peu aptes, changement dans la stratégie financière de la part des actionnaires, etc.). Parmi les plus emblématiques, El Tejar a été repris en 2014 par UAG [El Espectador, 2014] tandis qu'un Uruguayen contrôle, depuis mi-2015, $100 \%$ des actions d'ADP (Agronegocios del Plata), filiale de la méga-entreprise Los Grobo [El Observador, 2015]. Il resterait, entre autres, l'entreprise MSU et des producteurs argentins qui se sont installés sur quelques centaines ou milliers d'hectares, originaires souvent de la région de Córdoba.

Par ailleurs, des superficies sont entre les mains de sociétés transnationales dont l'origine des capitaux est étatsunienne ou internationale. Certaines (Agroterra, Noble Grain, Louis Dreyfus Commodities, Cargill, Glencore, Cereoil, etc.) fournissent au producteur les intrants nécessaires et peuvent déléguer les travaux agricoles. En revanche, elles se chargent de la commercialisation. D'autres (Kilafen, Adecoagro SA, Garmet du Groupe Perez Companc, etc.) possèdent les 
terres et font produire. Quelques-unes, dont les capitaux sont européens, investissent dans de grandes exploitations et les modernisent, à des fins productives (par exemple, Ingleby Farms\&Forests) ou spéculatives (Campos orientales de Pergam Finance).

Enfin, des entreprises uruguayennes fonctionnent avec des fonds d'investissement étrangers (exemple d'UAG). D'autres comme FADISOL, Barraca Erro SA (toutes deux ayant démarré avec des activités de commercialisation de produits agricoles) et des coopératives (Copagran, Calmer et Unión Rural de Flores, principalement) peuvent gérer de grandes superficies selon des modèles similaires à celui du pool. D’anciens métayers (Kent, Charbonnier, Campanella, Wornicov, etc.), après un temps de crise, produisent sur des terres en location (jusqu'à 20000 hectares pour certains d'entre eux) et qu'ils travaillent avec le matériel de l'entreprise de services agricoles qu'ils ont créée.

Finalement, du fait du rôle de «los argentinos »dans l'intensification et la spécialisation productive, et de l'évolution des entreprises uruguayennes, 5,8 millions de tonnes de grains ont été obtenues en 2013-2014 sur près de 1,6 million d'hectares (figure 3). Le soja arrive en tête avec plus de 3 MT et 1,3 Mha, le blé ensuite, et le maïs enfin. Dix ans plus tôt, 377000 tonnes de soja avaient été obtenues sur 247000 hectares.

Figure 3 -Évolution de la production et de la surface semée de soja et de la production et de la surface agricole totale en Uruguay (2000-2014)

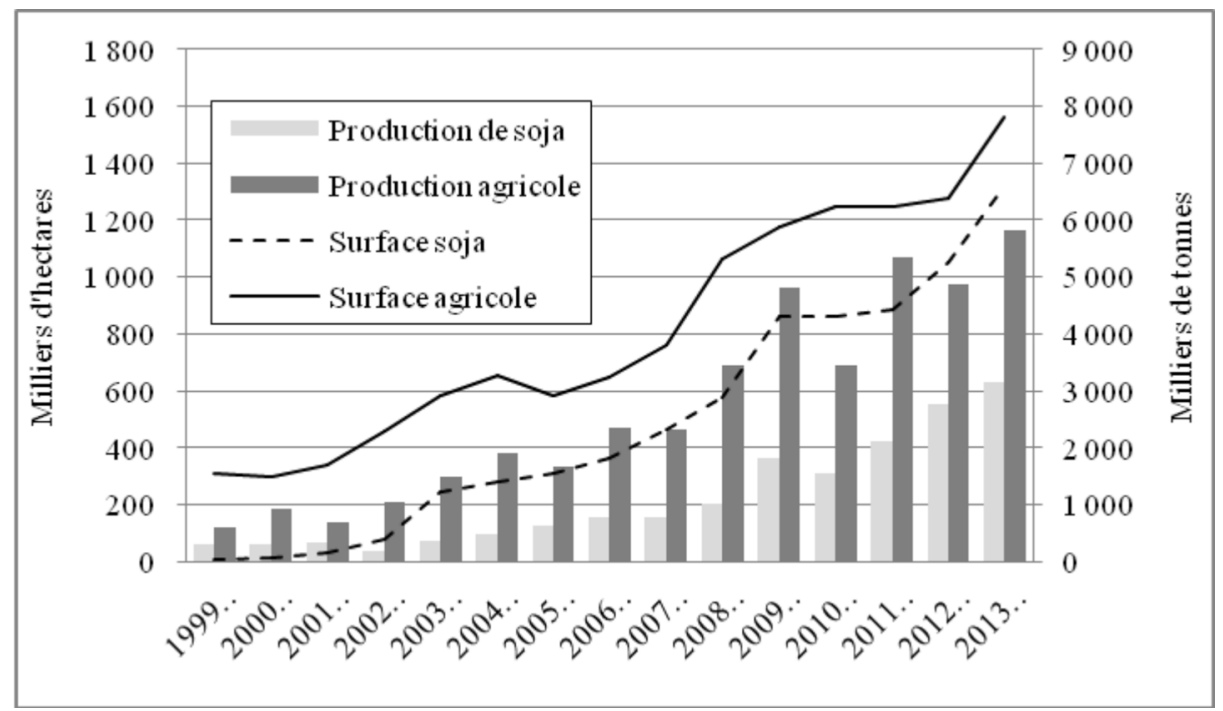

Source : ministère de l'Agriculture et de l'Élevage uruguayen - Direction des statistiques (MGAP-DIEA).

L'augmentation des surfaces semées dans les départements de l'Ouest et du Centre (Paysandú, Río Negro, Soriano, Tacuarembó, Durazno) s'est faite au 
détriment de l'élevage, avec l'incorporation de terres parfois peu aptes pour l'agriculture (carte 2).

Carte 2 - Superficies semées en cultures d'été (soja, sorgho, maïs, tournesol) en 2010-11 et taux d'augmentation par département entre 1990 et 2010

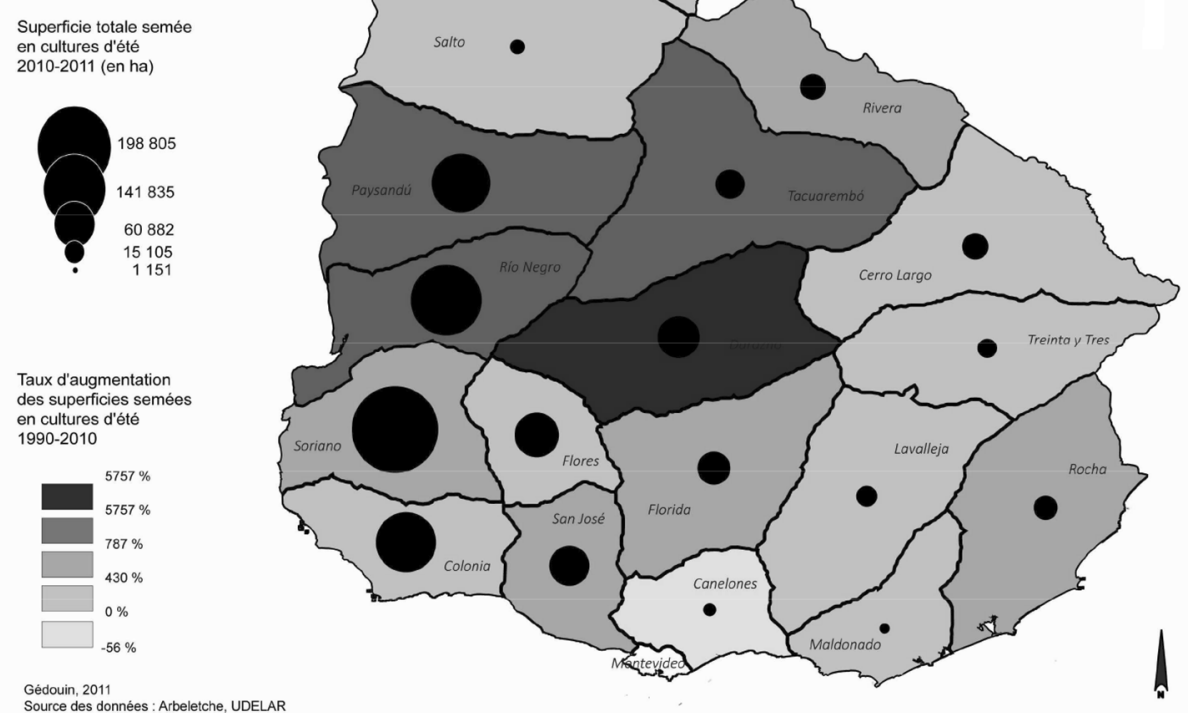

Source : carte élaborée par les auteurs.

Filière bois et cellulose : contrôle des plantations et de l'industrie de transformation principalement par des capitaux finlandais et chiliens

Au début des années 1990, une décennie avant le boom du soja, des acteurs industriels étrangers ont lancé la filière bois en Uruguay. Ils ont acheté des terres à de petits producteurs en difficulté (crises successives des années 1980 et 90), ce qui leur a permis de les vendre et de solder leurs dettes. La conséquence est leur départ pour des centres urbains, perçus comme des lieux de vie meilleurs, surtout pour les exploitants âgés, proches de la retraite. Depuis la moitié des années 2000, une autre modalité s'est imposée, appelée «producteurs associés» : des contrats de long terme (quelques dizaines d'années) peuvent lier entreprises et exploitants, ceux-ci restant propriétaires de leurs terres et étant engagés dans la production d'eucalyptus.

En 2012, les plantations d'eucalyptus et de pins atteignent 1 million d'hectares (figure 4). 
Figure 4 - Évolution de la surface cumulée en plantations sylvicoles en Uruguay, 1975-2012

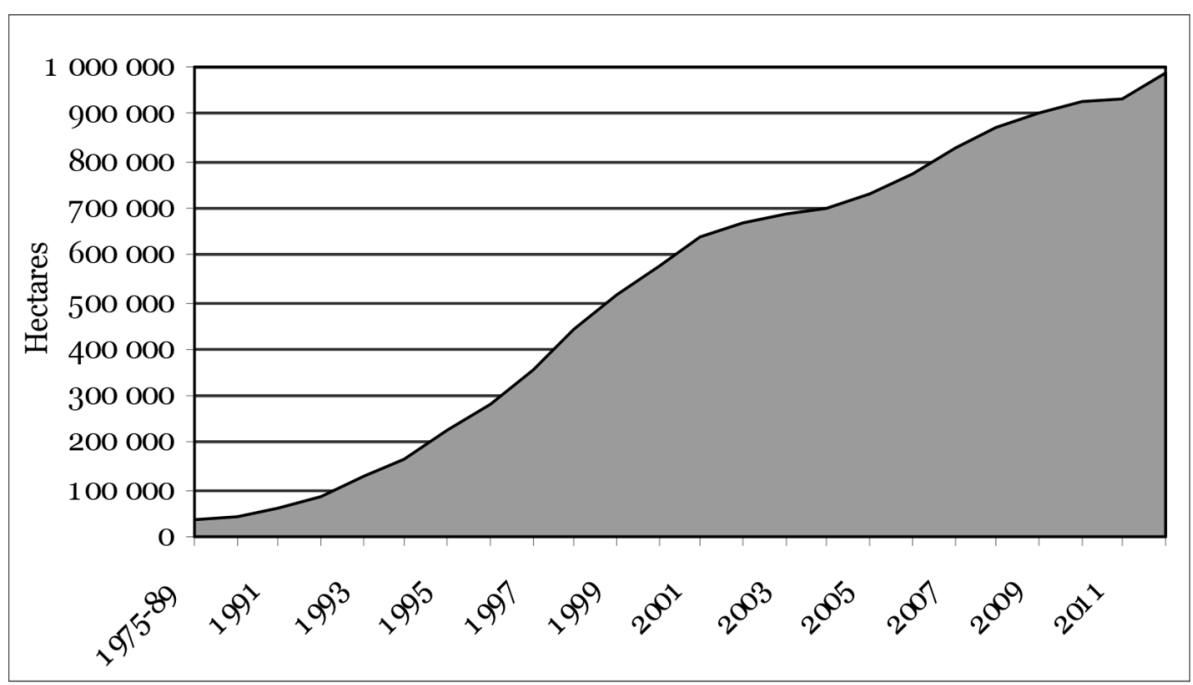

Source : ministère de l'Agriculture et de l'Élevage uruguayen - Direction générale des forêts (MGAP-DGF).

Après le développement précoce des plantations à l'Ouest (Départements de Paysandú, Río Negro), au Nord et au Centre (Départements de Rivera, Tacuarembó et Lavalleja), ce sont ceux de Treinta y Tres (à l'Est) et de Salto (au Nord) qui connaissent les plus fortes progressions dans les années 2000, au détriment de l'élevage (carte 3).

La finalité des plantations sylvicoles a évolué au cours du temps (figure 5). Si, au départ, elles étaient destinées surtout à la fabrication de chips, la cellulose en vue de la pâte à papier a pris le relais [Gautreau, 2014]. Le débouché énergétique sous forme de production d'électricité grâce à des chaudières est plus récent. Il prend son essor avec la volonté de valoriser les déchets d'une exploitation sylvicole devenue massive (branches, produits des coupes d'éclaircies, chutes de bois et sciure des scieries). Le «bois de feu » correspond à de vieilles plantations des années 1970 qui alimentent certaines industries locales et qui sont rarement possédées par des groupes transnationaux.

Quant aux acteurs étrangers, leur présence est écrasante. Trois groupes se partagent la quasi-totalité des plantations. Le premier d'origine finlandaise, UPM Forestal oriental (ex-Botnia), contrôle 135000 hectares plantés sur les 225000 hectares qu'il possède à l'Ouest du pays. Il produit chaque année 1,3 million de tonnes de pâte de cellulose dans le complexe industriel d'UPM situé à Fray Bentos, sur le fleuve Uruguay, et inauguré en novembre 2007. Forestal Oriental dirige les différentes étapes de l'activité sylvicole (plantation, récolte, transport). 
Carte 3 - Superficies plantées en 2013 en eucalyptus et en pins, et taux d'augmentation par département entre 1995 et 2013

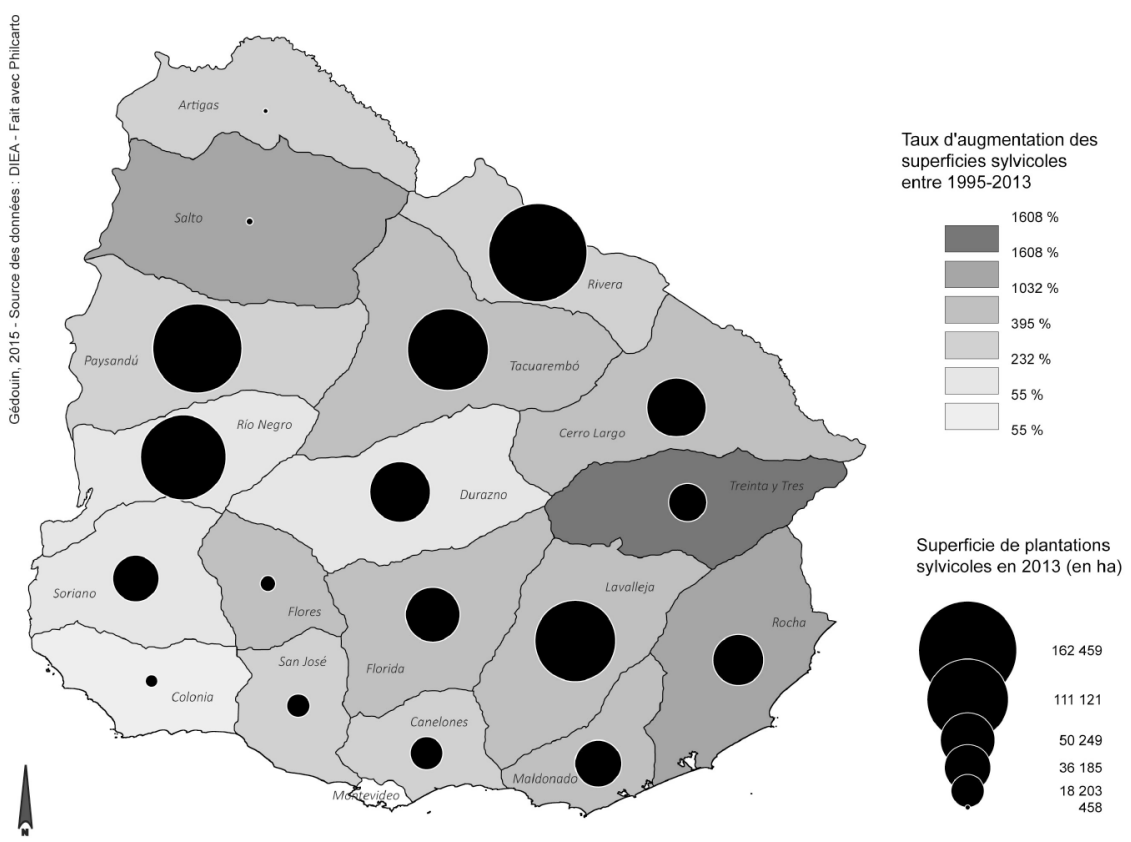

Source : carte élaborée par les auteurs.

Figure 5 - Évolution de la superficie sylvicole en fonction du produit final (2000-2014)

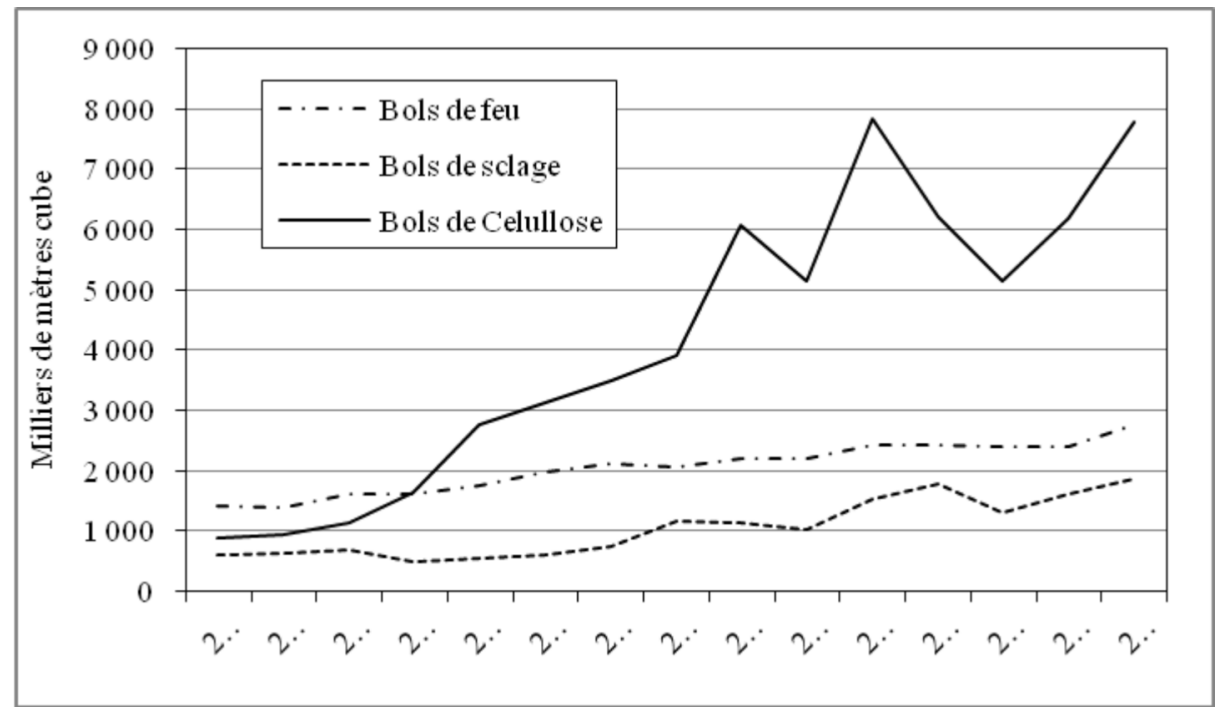

Source : ministère de l'Agriculture et de l'Élevage uruguayen - Direction des statistiques (MGAP-DIEA). 
Le deuxième groupe est Montes del Plata qui résulte d'une joint-venture entre Arauco (Chili) et Stora Enso (Suède-Finlande). Il possède 130000 hectares et en loue plus de 6000 hectares. 90 à 100000 sont plantés en eucalyptus, 33000 en pin. Le groupe a repris en 2009 les actifs vendus par Eufores, qui rassemblait le patrimoine agricole local du groupe espagnol ENCE, qui s'est retiré. Eufores avait orienté ses activités vers la production d'eucalyptus à des fins cellulosiques et de bois scié de qualité. Avec l'usine habilitée mi-2014 près du bourg de Conchillas, Montes del Plata fabrique 1,3 million de tonnes de pâte de cellulose par an.

Le troisième acteur est l'entreprise Weyerhaeuser (États-Unis) qui dispose de 55000 hectares plantés sur 140000 hectares en propriété, et qui produit du contreplaqué dans son usine de Tacuarembó. Avec 14 millions d'hectares en Amérique du Nord, en Australie et en Nouvelle-Zélande, elle est l'une des plus grandes entreprises de l'industrie sylvicole au monde; elle travaille en partenariat avec des fonds d'investissement.

Ces trois géants de la chaîne de valeur sylvicole et papetière concentrent près de $40 \%$ de la superficie totale plantée en Uruguay. Avec les autres entreprises partenaires de fonds d'investissement internationaux, et des sociétés forestières chiliennes comme Cambiun, c'est plus de la moitié de l'aire totale du pays qu'elles contrôlent. Dernièrement, dans le cadre de mesures de responsabilité sociale, des essais de sylvo-pastoralisme ont été pratiqués.

La territorialisation en Uruguay de ces acteurs argentins et brésiliens, et dans une moindre mesure, chiliens, européens ou étatsuniens, a changé le secteur agricole. Le soja développé par les entreprises d'origine argentine s'est imposé au point que certains producteurs locaux, par hybridation, en ont adopté le modèle, à une autre échelle et avec des moyens moins importants. L'eucalyptus se déploie quant à lui sur de vastes plantations gérées par les géants mondiaux de l'industrie sylvicole et papetière. L'élevage bovin, enfin, voit la filière être remontée par les puissants acteurs brésiliens de l'abattage. La filière riz est également investie par des producteurs brésiliens, beaucoup venant de l'État voisin du Rio Grande do Sul aux conditions pédologiques et climatiques semblables. Le secteur de la malterie, enfin, est passé sous le contrôle de la filiale AmBev du groupe belgobrésilien InBev [Gabinete productivo, 2013].

Le contexte est similaire dans l'ensemble du Cône sud où ces acteurs sont nombreux, avec une échelle d'action parfois régionale : entreprises de production de soja d'origine argentine au Brésil, groupes forestiers chiliens, producteurs brésiliens de soja au Paraguay, groupes sucriers spécialistes des zones tropicales, éleveurs uruguayens dans le Chaco en Argentine et au Paraguay qui représente une nouvelle frontière agricole en Amérique du Sud). 


\section{Des conséquences multiples}

Avec la présence de ces acteurs étrangers dans l'agriculture de leur pays, les producteurs uruguayens ont un autre rapport au foncier, l'envolée de sa valeur perturbant les schémas productifs classiques et altérant sa fonction patrimoniale. Les modes de production sont bouleversés, tendus vers l'intensification, et la fonction productive des ressources locales est renforcée. Les profils sociaux évoluent, les postes de travail offerts par les entreprises étrangères poussant à de nouvelles aspirations professionnelles. Par ailleurs, l'agriculture uruguayenne est davantage reliée à celle des pays voisins.

\section{Envolée des prix de la terre}

La forte pression sur le foncier favorise une augmentation de sa valeur. Côté location, le pool de siembra argentin a proposé rapidement un loyer une fois à une fois et demi plus élevé que le revenu net produit par l'exploitation directe de la terre ( $c f$. les entretiens réalisés). Par ailleurs, si la location de terre existait déjà, avec souvent des accords tacites entre locataire et propriétaire pour plusieurs années, la relation est maintenant plus formelle avec des contrats signés, et ce pour une durée moins longue qu' auparavant. Beaucoup d'exploitants se sont ainsi laissés tenter et ont loué une partie de leurs terres par contrat, pour une à quelques années. Cela a été en particulier le cas de propriétaires âgés, sans perspective de retraite décente, souvent sans enfant prêt à reprendre l'exploitation, et de jeunes pluriactifs voyant dans la location un moyen de valoriser leur terre.

Côté achat de terres, le nombre de transactions a été élevé en 2001-2002, au moment de la crise financière (figure 6). Certains ont vendu des hectares pour avoir des liquidités, d'autres en ont acheté pour placer leur épargne qui se dévalorisait. Au début, le prix moyen par hectare est resté stable, vraisemblablement en raison de l'équilibre entre l'offre et la demande de terres. Il a ensuite rapidement augmenté, soutenu par la demande de nombreux petits investisseurs revenant à des stratégies traditionnelles d'achat. De plus, l'arrivée des pools de siembra argentins offrant un excellent prix à la location a participé à la hausse du prix des terres les plus fertiles, de 500 dollars l'hectare en moyenne en 2008 à 3 500-4 000 dollars en 2013 (voir beaucoup plus pour certaines parcelles ou exploitations).

Ces niveaux, inédits pour les producteurs gauchos uruguayens, accroissent la valeur de leur bien foncier bien plus que ce que sa valorisation productive pourrait procurer. Beaucoup ont ainsi pu survivre, certains étant moins précarisés et étant de potentiels rentiers. En même temps, des exploitations sont vendues par les plus fragiles ou par ceux qui sont tentés par une vie en ville, a priori plus facile. Au-delà des acheteurs étrangers, des Uruguayens contribuent aussi à l'animation du marché foncier national [Piñeiro, 2012], qui entre dans le cadre d'une dynamique qui touche l'ensemble de l'Amérique latine [Borras et al., 2012]. 
Figure 6 - Superficie foncière vendue par an entre 2000 et 2013 et prix moyen par hectare en dollars

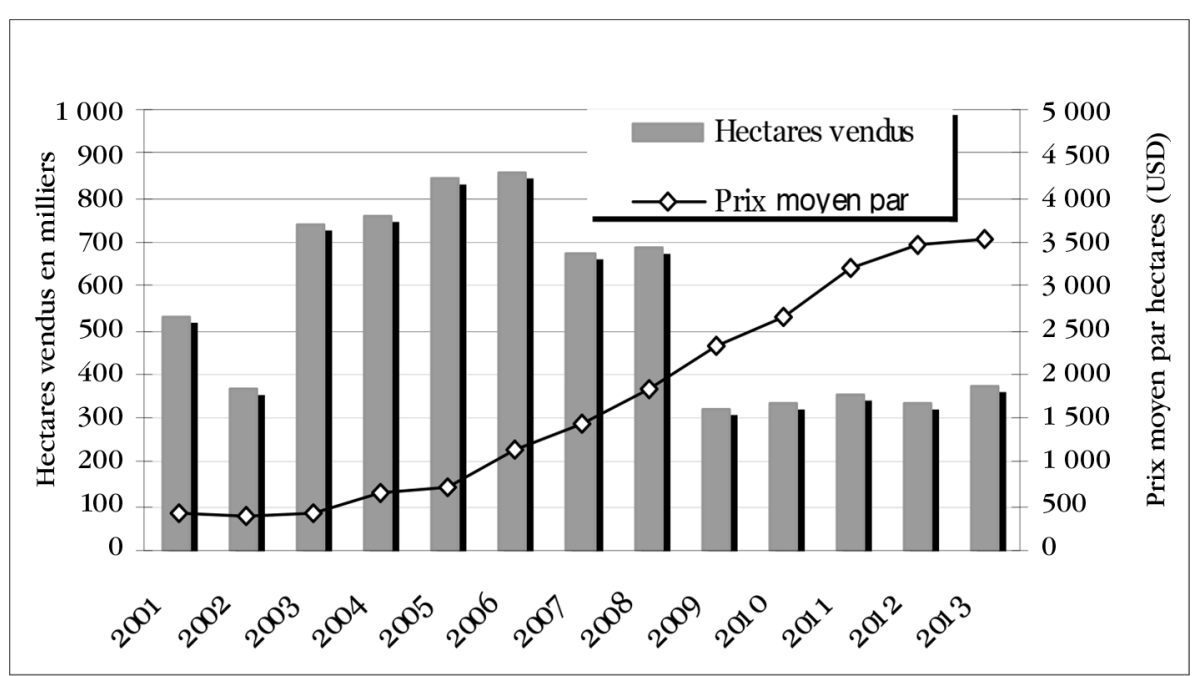

Source : ministère de l'Agriculture et de l'Élevage uruguayen - Direction des statistiques (MGAP-DIEA).

En combinaison avec d'autres facteurs comme l'endettement ou la fragilité de la structure productive, l'un des résultats de cette cession accélérée de terres à des acteurs uruguayens ou étrangers est la disparition de 12000 exploitations agricoles entre 2000 et 2010 (une baisse d'environ $20 \%$ ), en général des petits et moyens producteurs (ministère de l'Agriculture et de l'Élevage uruguayen - MGAP).

La concentration foncière se manifeste en particulier dans la filière sylvicole, du fait de l'achat de terres dès les années 1990 par les trois géants du secteur. De plus, comme en Argentine, dans le cas des grains, plusieurs exploitations agricoles peuvent être travaillées par une seule entreprise. Elle prend en charge les décisions relatives à la production, le propriétaire des terres étant peu impliqué et comptant sur la rente négociée avant récolte. Il y a dans ce cas un processus de concentration productive.

\section{Délégation des activités et approche entrepreneuriale et technique du métier}

La sous-traitance des travaux agricoles s'est généralisée sur la base de relations contractuelles formalisées entre le producteur et les acteurs qui assurent, du fait de la possession des machines, le semis, les traitements phytosanitaires ou la moisson. Il y a déverticalisation des liens entre l'entreprise agricole et ses associés, l'engagement par contrat organisant des relations plus réticulaires que hiérarchiques.

Par ailleurs, l'homogénéisation et la standardisation des étapes des processus de production sont accompagnées par une grande spécialisation de chacune d'entre elles. Le recours à des moyens technologiques tendant à augmenter la productivité, 
à limiter les coûts de transaction et à assurer la qualité industrielle des productions est plus fréquent. De même, l'utilisation plus systématique d'intrants et d'innovations va de pair avec la complexification de la gestion de l'ensemble des activités, de la production à la commercialisation. Cela suppose des compétences accrues, une division du travail et un redimensionnement de l'échelle des opérations, ainsi que des innovations organisationnelles pour limiter les coûts d'administration.

\section{Implications sociales}

En plus du départ des agriculteurs les plus faibles, l'avancée de l'agrobusiness globalisé modifie le marché du travail. Si les ouvriers agricoles moins préparés n'ont d'autre recours que l'exode rural, les autres accèdent à des emplois caractérisés par leur stabilité, leur caractère professionnel et des salaires considérés comme raisonnables à corrects. Ingénieurs agronomes, conseillers techniques, chauffeurs spécialisés, fournisseurs d'intrants, etc. : autant d'activités valorisées permettant de faire reconnaître des compétences et des expériences accumulées de la formation universitaire (agronomie, administration, etc.) aux différents postes occupés. Ces jeunes professionnels établis dans les bourgs, dotés de pick-up et des outils de la communication mobile, revendiquent un mode de vie urbain et gèrent des trajectoires professionnelles plus ouvertes sur le monde. La consolidation de cette strate sociale en milieu rural, plus précisément, dans les bourgs et petites villes, s'inscrit dans l'évolution de la population dans son ensemble. L'Uruguay présente depuis longtemps des indicateurs sociaux parmi les plus hauts en Amérique du Sud. Les réformes du Code du travail et l'effort éducatif des gouvernements de gauche à partir de 2005 ont été décisifs pour créer une aspiration à une amélioration des conditions de vie. Les professionnels de l'agriculture entrepreneuriale désirent donc des cadres de vie modernes et, souvent, la petite ville n'offre pas les services espérés, notamment pour le conjoint et le reste de sa famille. Les mobilités sont alors fréquentes entre l'espace agricole de travail et l'espace urbain de vie (ville moyenne à grande).

\section{Relations avec l'Argentine et le Brésil}

De tout temps, la marge de manœuvre de l'Uruguay face à ses deux grands voisins a été source de friction. Preuve en est le conflit avec l'Argentine à propos de l'installation des usines de pâte à cellulose sur le Rio Uruguay, ou l'impossibilité pour l'Uruguay de créer des liens commerciaux avec l'Alena ou l'Union européenne sans avoir l'approbation de ses voisins. Or, en s'agrégeant à la dépendance du tourisme uruguayen vis-à-vis de ses voisins, la présence des entreprises argentines et brésiliennes dans les secteurs agricole et agro-industriel, complique la possibilité pour l'Uruguay d'exercer une politique commerciale indépendante. Les interrelations accentuées par la globalisation se manifestent ici de manière presque caricaturale : des entreprises des pays voisins utilisent les ressources naturelles de l'Uruguay pour fournir des marchés extracontinentaux. Par ailleurs, les 
acteurs du secteur agricole soulignent les impacts de leur présence en termes d'incitation à la modernisation et à l'intensification des processus productifs.

\section{Conclusion}

Depuis deux décennies, l'Uruguay accueille des cultures, telles que le soja et l'eucalyptus, impulsées par des acteurs étrangers (Sud-Américains et autres). Elles ont transformé l'agriculture, les espaces ruraux et l'économie nationale dans son ensemble par la dynamique suscitée, l'offre d'emplois qualifiés, la diffusion d'innovations organisationnelles et productives, et la rapide augmentation des exportations de commodities. Le gouvernement uruguayen et les entreprises étrangères défendent le caractère vertueux des mutations du monde rural, des modes de production en agriculture et dans l'élevage, et la contribution du secteur à l'équilibre de la balance commerciale. La diversification des productions destinées à l'exportation, avec la filière sylvicole, est censée rendre l'économie moins vulnérable aux fluctuations des commodities. L'amélioration de la qualité de la viande permettrait d'atteindre des marchés de niche moins susceptibles d'effondrement. La «mise à niveau » en termes de productivité (des ressources, des structures, etc.) est telle qu'elle rend plus résilients les systèmes, censés pouvoir s'adapter à un panorama international mouvant. Mais ces constats en apparence positifs cachent une réalité complexe. Des transformations profondes bousculent sur un pas de temps court (quelques années) le rapport à la terre et au métier d'éleveur qui fonde l'identité des producteurs uruguayens et de la société gaucha. Se pencher sur les effets d'hybridation chez les producteurs qui ne sont pas soumis à l'influence des groupes étrangers est une piste à suivre.

Si ces entreprises établies en Uruguay revendiquent leur origine sud-américaine, ce critère d'appartenance à la région joue à la marge, les impératifs économiques dépassant vite les anecdotes identitaires ou footballistiques. Bien plus significatif est le déplacement du centre de gravité de la gouvernance globale du monde vers l'Asie, avec la prise de participation d'acteurs financiers chinois, par exemple. Les pays pré-émergents du Sud-Est asiatique et du Moyen-Orient imposent progressivement leurs propres normes, comme l'ont fait la Chine et le Brésil au tournant du siècle dernier. Au même titre que l'automobile, l'informatique, les loisirs, etc., l'agrobusiness globalisé est inscrit dans cette dynamique, comme le montrent les filières viandes et soja, ou l'industrie papetière, qui sont en plein développement en Uruguay.

\section{Bibliographie}

Arbeletche P., Carballo C. [2010], «La expansión agrícola en Uruguay. Algunas de sus principales consecuencias », Revista de desarrollo rural y cooperativismo agrario, $\mathrm{n}^{\circ} 12$, p. 7-19. 
Arbeletche P., Coppola M., Paladino C. [2012], « Análisis del agro-negocio como forma de gestión empresarial en América del Sur : el caso uruguayo », Agrociencia, n 2, p. 110-119.

Arbeletche P., Gutierrez G. [2010], « Crecimiento de la agricultura en Uruguay. Exclusión social o integración económica en redes », Revista pampa, nº 6, p. 113-138.

BARRÁN J.-P., NAHUM B. [1967-1977], Historia rural del Uruguay moderno, Montevideo, Banda oriental.

Batello C., Berretta E.J., Hodgson J., Kothmann M., Li X., McIvor J., Milne J., Morris C., Peeters A., Sanderson M., The forage and grazing terminology committee [2011], " An international terminology for grazing lands and grazing animals », Grass and forage science, vol. 66, $\mathrm{n}^{\circ} 1$, p. 2-28.

BIRF-FAO [1951], Recomendaciones para el desarrollo agrícola del Uruguay : informe de la misión técnica auspiciada por el Banco internacional de reconstrucción y fomento y la FAO a petición del gobierno de la República oriental de Uruguay, Montevideo, Imprenta nacional.

Bisang R., ANLló G., CAMPI M. [2010], « Organización del agro. La transición de un modelo de integración vertical a las redes de producción agrícola », in RECA L.G., LEMA D., FOOD C., El crecimiento de la agricultura argentina. Medio siglo de logros y desafíos, Buenos Aires, FAUBA, p. 231-254.

Bittencourt G., Carracelas G., Reig N. [2011], « Determinantes y efectos de la inversión extranjera directa en la industria frigorífica uruguaya », Documento de trabajo, n ${ }^{\circ} 29$, Departamento de economía, Facultad de ciencias económicas y de administración, UdelaR.

Bittencourt G., Carracelas G., Doneschi A., Reig N. [2009], « Tendencias recientes de la inversión extranjera directa en Uruguay », Documento de Trabajo, $\mathrm{n}^{\circ} 27$, Departamento de economía, Facultad de ciencias económicas y de administración, UdelaR.

Borras JR S.M., KAY C., Gómez S., WiLKInSON J. [2012], « Land grabbing and global capitalist accumulation : key features in LatinAmerica », Canadian journal of development studies, vol. $33, n^{\circ} 4$, p. 402-416.

Buainain A.M., Alves E., Silveira J.M., Navarro Z. [2014], O mundo rural no Brasil do século 21. A formação de um novo padrão agrário e agrícola, Brasilia D.F., Embrapa, Brasil.

Clasadonte L., de Vries E., Trienerens J., Arbaletche P., Tourrand J.-F. [2013], « Network companies : a new phenomenon in South American farming », British food journal, vol. $115, \mathrm{n}^{\circ} 6$, p. 850-864.

EL ESPECTADOR [2014], «El Tejar: aun quedan definir algunos detalles de acuerdo », El espectador, 26 février : http://www.espectador.com/economia/285627/el-tejar-aun-quedan-definiralgunos-detalles-de-acuerdo (page consultée le 11 avril 2015).

El OBSERVADOR [2012], «Ofensiva financiera de los frigoríficos », El observador, 16 février: http://www.elobservador.com.uy/ofensiva-financiera-los-frigorificos-n218944 (page consultée le 11 avril 2015).

El OBSERVADOR [2013], « Brasilera Minerva anuncia más adquisiciones en Uruguay », El observador, 5 novembre 2013 : http://www.elobservador.com.uy/brasilera-minerva-anuncia-masadquisiciones-uruguay-n264365 (page consultée le 11 avril 2015).

El OBSERVADOR [2015], «Marcos Guigou y socios compraron participación de los Grobo en ADP », El observador, 31 juillet 2015 : http://www.elobservador.com.uy/marcos-guigouy-socios-compraron-participacion-los-grobo-adp-n665215 (page consulté le 30 septembre 2015). 
ERnSt O., SiRI-PRIETo G. [2012], La Agricultura en Uruguay : su trayectoria y consecuencias, II Simposio Nacional de Agricultura, Montevideo, Uruguay, p. 149-163.

Errea E., Peyrou J., Secco J., Souto G. [2011], Transformaciones en el agro uruguayo. Nuevas instituciones y modelos de organización empresarial, Montevideo, Universidad Católica del Uruguay.

Finch H. [1980], Historia económica del Uruguay contemporáneo 1870-2000, Montevideo, Banda oriental.

Gabinete PRoductivo [2013], «Inversiones de Brasil en Uruguay », Montevideo, República Oriental del Uruguay: http://gp.gub.uy/sites/default/files/documentos/inversiones_brasilenas_en_uruguay_1.pdf (page consultée le 11 avril 2015).

Garavaglia J. C. [2000], Les Hommes de la Pampa. Une histoire agraire de la campagne de Buenos Aires, 1700-1830, Paris, Maison des sciences de l'homme.

GAUTREAU P. [2014], Forestación, territorio y ambiente : 25 años de silvicultura transnacional en Uruguay, Brasil y Argentina, Montevideo, Trilce.

GuiBERT M. [2013], «Les formes associatives de production agricole en Argentine et en Uruguay : entre territoire et réseau ? », Études rurales, no 191, p. 77-90.

Guibert M., Grosso S., Arbeletche P., Bellini M.-E. [2011], «De Argentina a Uruguay: espacios y actores en una nueva lógica de producción agrícola », Pampa, suplemento especial temático, $\mathrm{n}^{\circ} 7$, p. 13-38.

LiTRE G. [2010], Os gaúchos e a globalização : vulnerabilidade e adaptação da pecuária familiar no pampa do Uruguai, Argentina e Brasil, Tese de doutorado, Centro do desenvolvimento sustentável, universidade de Brasília- IHEAL/CREDAL, Paris III, Sorbonne-nouvelle.

Moraes M.I. [2008], La Pradera pérdida. Historia y economía del agro uruguayo : una visión de largo plazo, 1760-1970, Montevideo, Linardi y Risso.

MoralEs H. [2007], Évaluation des conséquences de décisions stratégiques en élevage à l'herbe en Uruguay. Une approche par les systèmes multi-agents (SMA), thèse de doctorat, ABIES/ AgroParisTech, Paris, France.

Morales H., Homem V., Champredonde M., Tourrand J.-F. [2011], « South American Livestock Farming Expansion. The long Way to Sustainability », in Kammili T., Hubert B., TOURRAND J.-F. (dir.), A paradigm shift in livestock management : from resource sufficiency to funcional integrity, Proceedings of a workshop at the XXI Grassland/VIII Rangeland International Congress, June $29^{\text {th }}$ - July $5^{\text {th }} 2008$, Hohhot, China, Lirac, France, Cardère, p. 73-85.

PIÑEIRo D. [2012], «Land Grabbing : concentration and "foreignisation" of land in Uruguay », Canadian journal of development studies, vol. 33, $\mathrm{n}^{\circ} 4$, p. 471-489.

Requier-Desjardins D., Guibert M., BüHLER E. A. [2014], «La diversité des formes d'agricultures d'entreprise au prisme des réalités latino-américaines », Économie rurale, ${ }^{\circ}{ }^{3} 44$, p. $45-60$.

Saravia A., Morales H., Gédouin M., Tourrand J.-F. [2014], «Escenarios futuros para la ganadería y sus motores de cambio : desde la visión de los actores », II Congreso de Ciencias Sociales Agrarias "Enfoques y Alternativas para el Desarrollo Rural", Facultad de Agronomía, Universidad de la República, 6 al 8 de agusto de 2014, Salto, Uruguay.

TAMBler A. [2014], « Recaudación y presión fiscal en el agro », in Ministerio de Ganadería, Agricultura y Pesca, Anuario 2014 OPYPA, Montevideo, Uruguay, La Imprenta, p. 309-315. 
Tourrand J.-F., Vaz V., Veiga J.-B., Quanz D., Poccard-Chapuis R. [2013], « Pionniers d'Amazonie : une vision particulière de l'incertitude », in ANCEY V., Avelange I., Dedieu B. (dir.), Agir en situation d'incertitude, Paris, QuAE, p. 213-228.

URUGUAY XXI [2014], Inversión extranjera directa en Uruguay, Montevideo: www.uruguayyxxi.gub.uy (page consultée le 11 avril 2015).

Vassallo M. (dir.) [2012], Dinámica y competencia intrasectorial en el agro. Uruguay 2000-2020. La visión de los actores, Montevideo, Uruguay, Ediciones Universitarias.

\section{Webgraphie}

INSTITUT NATIONAL DES VIANDES (INAC), www.inac.gub.uy (page consultée le 4 avril 2015).

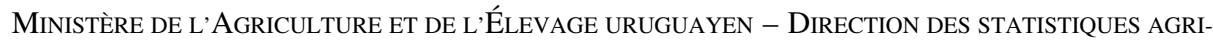
COLES (MGAP - DIEA), www.mgap.gub.uy/portal/page.aspx?2,diea,diea-principal, O, es, 0 (page consultée le 4 avril 2015).

Ministère de L'Agriculture et DE L'ÉleVAGE - DireCtion GÉNÉRAle des ForÊTS (MGAP Dirección General Forestal) : http://www.mgap.gub.uy/portal/page.aspx?2, dgf, dgf-principal, O, es, 0 (page consultée le 4 avril 2015). 